\title{
Delay and Reversal of Chloroplast Senescence Structure in the Dicot Nicotiana rustica and the Monocot Triticum aestivum.
}

\author{
H.A. Zavaleta-Mancera*, and L.F. Jimenez-Garcia.**. \\ * Colegio de Postgraduados, Instituto de Recursos Naturales, Montecillo, 56230, Edo. Mex. Mexico. \\ ** Universidad Autonoma de Mexico, Facultad de Ciencias, C.U. , 04510 D.F., Mexico.
}

Ultrastructure of senescing leaves has been investigated in several species of monocots and dicots recognising the chloroplast as the earliest organelle to change in leaf senescence [1]. Several experiments have demonstrated that cytokinins have effects on chlorophyll biosynthesis, chloroplast differentiation and structure at early stages of leaf development. Addition of cytokinin to etiolated leaves or cotyledons enhances subsequent development of etioplast into chloroplasts, especially promoting grana formation and an increases of the rate of chlorophyll synthesis [2]. External applications of cytokinins to detached or attached leaves delays senescence and in some particular cases it can promote regreening in dicots [3 and 4]. Are the dicot- chloroplasts representing a higher status of organelle plasticity in contrast to the monocot-chloroplast?. Is this an indication of evolutionary adaptation of survival to extreme conditions or death? . The present study monitored chloroplast ultrastructure of the dicot Nicotiana rustica L and the monocot Triticum aestivum L. leaves during: normal senescence, delay and reversal of senescence promoted by citokinin treatment.

Nicotiana rustica. A series of progressively senescing leaves of the sixth node were studied; green $(\mathrm{G})$ 10 weeks old, senescent 1 (S-1) 14 weeks old and senescent 3 (S-3) 15 weeks old. Three different stages of regreening (6, 13 and 20 days) were obtained from decapitated shoots treated with BAP 10-4 M [4]. Triticum aestivum segments from the middle part of mature second leaves were incubated in sterile humid chambers containing a solution of BAP 10-4 $\mathrm{M}$ or water as a control. They were incubated in dark conditions to promote senescence, during 2, 4, and 6 days. Nicotiana tissue was prefixed with $5 \%$ glutaraldehyde- $2 \%$ paraformaldehyde in $67 \mathrm{mM}$ cacodylate buffer $\mathrm{pH} \mathrm{7.2,} \mathrm{postfixed} \mathrm{in} 1 \%$ osmium tetroxide and embedded in LR White resin. Triticum tissue was prefixed with $6 \%$ glutaraldehyde in Sorensen s phosphate buffer $\mathrm{pH} 7.2$, post fixed in 1\% osmium tetroxide and embedded in Epon resin. Ultra thin sections were double stained with $2 \%$ uranyl acetate and lead citrate [5].

T. aestivum leaves treated with BAP showed a significant delay of senescence. After 6 days in the dark, BAP treated tissue lost only $36 \%$ of its initial total chlorophyll content while the water treated tissue (control) lost $81 \%$ of its formal chlorophyll. Green chloroplast, before treatment (day 0) showed well differentiated grana thylakoid and intergrana regions, associated to small plastoglobuli (Fig. 1A). After 4 days, water treated leaves (control) showed signs of senescence, disassemble of grana thylacoids, increase in size and number of plastoglobuli (Fig. 1.B). Two days later (6 days) no intact chloroplasts were observed. In contrast 6 days BAP treated leaves preserved their chloroplast integrity, stroma opacity and grana arrangement, presenting a similar structure to the green chloroplast (Fig. 1.C).

External applications of BAP to $N$. rustica leaves not only delayed the chloroplast senescence, but also promoted the reversal of chloroplast senescence in terms of pigments, protein and structure. Experiments carried out on reversal of three stages of senescence (S-1, S-2 and S-3) indicated that the redifferentiation capacity of gerontoplasts decreased dramatically at late senescence (S-3), after the leaf had lost more than $87 \%$ of its formal chlorophyll. The gerontoplast with the capacity to reverse (S-1), was round, containing large plastoglobuli, with a $17 \%$ of plastid area as plastoglobuli, thylakoid membranes distended and swollen, forming individual flat sheets (Fig. 2.B). In contrast S-3 gerontoplasts can not regreen, they do not contain any internal membranes and the plastid is full of plastoglobuli with some sings of plastid envelope disintegration. During the reversal of senescence the plastid recovered progressively its shape, population of polyribosomes, new thylakoids started to form grana stacks 
preferentially at the peripherical region and the starch grains were consumed. The $\%$ of plastid area as plastoglobuli decreased from $16 \%$ to $13 \%$. The persistence of large plastoglobuli in the regreened plastid is a mark of its senescent origin, giving evidence of the re-diferentiation phenomena.

Our results indicated that integrity of chloroplast structure and the capacity to re-differentiate during senescence was better preserved in the Dicot N. rustica than in the Monocot T. aestivum. Monocot leaves grow with a basal meristem and hence form a developmental gradient, the base of the leaf is younger and contain mainly proplastids while the oldest cells are found close to the tip [6]. In contrast dicot leaves grow with a marginal and laminar meristem where differentiation of chloroplasts occur in a more uniform manner. The strategy to face extreme conditions that promotes senescence in Dicots appear to be more related to the genetic plasticity of their gerontoplasts to re-differentiate into re-greened chloroplast while in Monocots the presence of a basal meristem could provide a new population of chloroplasts when the gerontoplasts of the middle and apex of the leaf were dead [7].
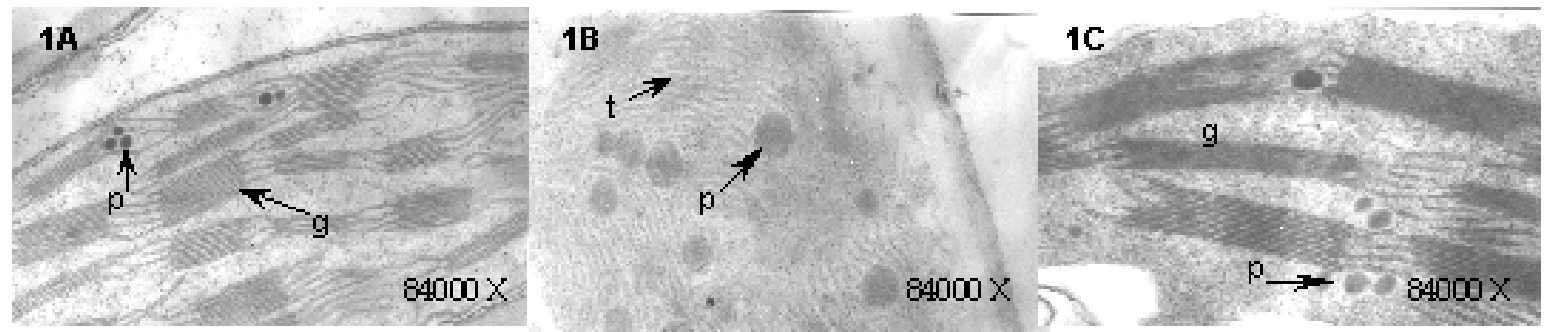

Fig. 1. Delay of senescence of Triticum aestivum chloroplasts. A) Green chloroplast from a mature green leaf, B) Senescent chloroplast from a 4 days water treated leaf, C) Chloroplast from a 6 days BAP treated leaf showing preserved grana and stroma structure. p:plastoglobuli, g: grana t: swollen thylakoids.
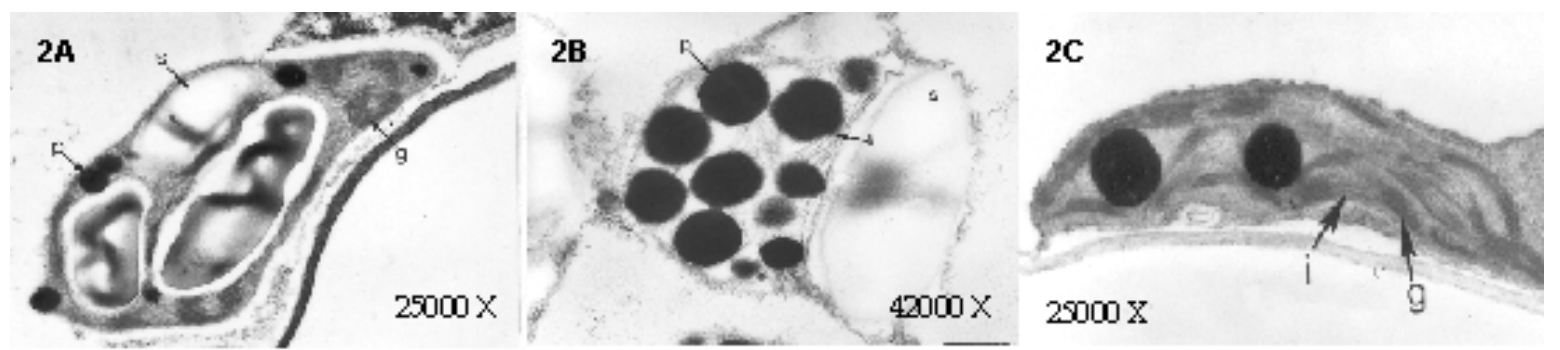

Fig. 2. Reverse of senescence of Nicotiana rustica chloroplasts. A) Green chloroplast from a mature green leaf, B) Senescent chloroplast, (S-1) from a yellow leaf C) Regreened chloroplast, from a 20 days BAP treated leaf, showing recovery of the grana and stroma structure lost during senescence. g: grana, $\mathrm{p}$ : plastoglobuli, s: starch grain, t: swollen thylakoid.

\section{References.}

[1] U.C. Biswall and B. Biswall., International Review of Cytology. 113 (1988) 271.

[2] J. Chory et al., Plant Physiology. 104 (1994) 333.

[3] S. Amasino and R.M. Amasino. Science. 270 (1995) 197.

[4] H.A. Zavaleta et al., Journal of Experimental Botany. 50 (1999) 1677.

[5] E.S. Reinolds. Journal of Cell Biology. 139 (1963) 208.

[6] U.C.Vothknecht and P. Westhoff. Biochimica et Biophysica Acta 1541 (2001) 91.

[7] This research was suported by the Consejo Nacional de Ciencia y Tecnología, CONACYTMexico, proyecto I37585-B. 